\title{
Ancient Roots of Today's Emerging Renaissance in Psychedelic Medicine
}

\author{
Daniel R. George ${ }^{1}$ (D) Ryan Hanson ${ }^{2} \cdot$ \\ Darryl Wilkinson ${ }^{3}$ - Albert Garcia-Romeu ${ }^{4}$
}

Accepted: 25 August 2021/Published online: 2 September 2021

(C) The Author(s), under exclusive licence to Springer Science+Business Media, LLC, part of Springer Nature 2021

\begin{abstract}
An international ban on psychedelics initiated by the United Nations' Convention on Psychotropic Substances in 1971 restricted the clinical use of these ancient psychoactive substances. Yet, in an era marked by rising mental health concerns and a growing "Deaths of Despair" epidemic (i.e., excess mortality and morbidity from suicide, drug overdose, and alcoholism), the structured psychedelic use that has long been a part of ritual healing experiences for human societies is slowly regaining credibility in Western medicine for its potential to treat various mental health conditions. We use a historical lens to examine the use of psychedelic therapies over time, translate ancient lessons to contemporary clinical and research practice, and interrogate the practical and ethical questions researchers must grapple with before they can enter mainstream medicine. Given the COVID-19 pandemic and its contributions to the global mental health burden, we also reflect on how psychedelic therapy might serve as a tool for medicine in the aftermath of collective trauma. Ultimately, it is argued that a "psychedelic renaissance" anchored in the lessons of antiquity can potentially help shift healthcare systems — and perhaps the broader society-towards practices that are more humane, attentive to underlying causes of distress, and supportive of human flourishing.
\end{abstract}

Daniel R. George

Dgeorge1@pennstatehealth.psu.edu

1 Department of Humanities, Penn State Milton S Hershey Medical Center, Penn State College of Medicine, Hershey, PA 17033, USA

2 Anesthesiology Institute, Cleveland Clinic, Cleveland, OH, USA

3 Department of Religion, Dartmouth University, Hanover, NH, USA

4 Center for Psychedelic and Consciousness Research, Department of Psychiatry and Behavioral Sciences, Johns Hopkins School of Medicine, Baltimore, MD, USA 
Keywords Mental health · Psychedelic medicine - Health humanities ·

Ethics · Clinical medicine

In 2008, archaeologists excavating a cave in the Sora River valley in southwestern Bolivia discovered a 1,000-year-old ritual bundle formerly belonging to a shaman of the pre-Inca Tiwanaku civilization. Scrapings from the artifacts inside revealed traces of psychotropic compounds-benzoylecgonine (a metabolite of cocaine), bufotenine (an alkaloid in psychedelic snuff powder), psilocin (the active agent in magic mushrooms), and harmine and dimethyltryptamine (the active ingredients in the psychedelic tea known as ayahuasca) (Miller et al. 2019).

Use of psychoactive substances in healing practices is often associated with "shamanism", although the practice was so ubiquitous in the ancient world that it should effectively be considered a species "norm". Ritual use of drugs has often been associated with hunter-gatherer cultures across the globe-from the Americas, to Eurasia, to Australia, and Africa (Walter and Fridman 2004). However, there is no reason to believe that hunter-gatherers are the only societies in which psychoactive substances have been of central importance. Writing in the fourth century BC, Herodotus described ritual use of cannabis by the Scythians of the Pontic Steppe, a practice recently corroborated by archaeological analysis of braziers used in funerary rites (Ren et al. 2019). The ancient Greeks-and later the ancient Romans and agrarian societies in the near east and Minoan Crete-held the Eleusinian Mysteries, seasonal religious rites that included ritual ingestion of a psychoactive drink called kykeon, which evidence has shown may have included ergot fungi containing LSD-like psychedelic alkaloids (Samorini 2019; Wasson, Hofmann, and Ruck 1978; Ouvaroff 1817). Moreover, the soma drink described in the Rigveda clearly refers to a non-alcoholic psychoactive substance, although its precise identity is a matter of debate (Staal 2001). A related libation, hoama, is also prominent in the Avestas, the ancient scriptures of Zoroastrianism. Ancient American societies also appear to have embraced psychoactive ritualism to an exceptional degree, including those that were urban and made use of writing, such as the Maya (Dobkin de Rios 1974).

Indeed, while the materials discovered in the Bolivian cave were exceptional for their preservation and diversity, there is nothing remarkable about the ancient use of psychedelics per se. What is unusual is the active suppression of such substances, a late twentieth century global phenomenon led by the United States and United Nations (albeit with deeper roots in Christianity and the colonial Americas) (CarodArtal 2015). Today, some healers in Western countries still employ psychoactive plants, often illicitly due to the still-binding international bans on psychedelics initiated by the UN's Convention on Psychotropic Substances of 1971 (Nutt et al. 2013).

But in our current era marked by growing mental health concerns (Vahratian et al. 2021), prohibitions against compounds that have long played an integral role in mediating mental and spiritual health in human societies feel increasingly out-ofstep. Indeed, the early twenty-first century has not only ushered in rising depression 
and anxiety (Lim et al. 2018; Ettman et al. 2020), but also a crisis of "Deaths of Despair" (DoD)—excess mortality and morbidity from suicide, drug overdose, and alcoholism, especially among working class adults in mid-life with low educational attainment (Case and Deaton 2020, 2021; Brignone et al. 2020; George et al. 2021). These self-harming deaths, which are strongly linked to growing feelings of distress (Blanchflower and Oswald 2020), loss of social cohesion (Case and Deaton 2020; 2017; 2015; Marmot 2018; Scutchfield and Keck, 2017; Stein et al. 2017), isolation and disconnection from social institutions (Smeraldo et al. 2020), and loss of hope for the future, are estimated at 150,000 per year in the US. DoD are rendered even more socially damaging by distal factors such as access to handguns and lethal drugs (e.g., fentanyl) (Shiels et al. 2020; Well Being Trust 2021), that, taken together, have contributed to an annual downward trend in life expectancy in the US from 2015 to 2017, representing the longest sustained decline since 1915-1918 (Murphy et al. 2018).

While DoD were initially characterized as a phenomenon disproportionately affecting non-Hispanic white populations in economically-distressed regions, including the Midwestern "Rust Belt" and Appalachia (Case and Deaton, 2015; Dwyer-Lindgren et al. 2017), more recent evidence suggests that the crisis of rising despair-related mortality extends across racial, ethnic, and gender groups as well as geographic locales and generational cohorts (i.e., Millennials) (Sheils et al. 2020; Well Being Trust 2020; Gaydosh et al. 2019; Woolf and Schoomaker 2019; Case and Deaton 2021). So too have upticks in despair-related mortality recently been observed in other Western countries, including the UK, Canada, and Australia (Case and Deaton 2020). It seems that despair is proliferating in an era defined by globalization, deindustrialization, deunionization, and increasing automation, as well as historic recessions, record income and wealth inequality, prolonged wage decline, austerity cuts to social safety nets (Case and Deaton 2020), and now a global pandemic that has exacerbated these systemic failures (Vahratian et al. 2021). As structural conditions have broadly worsened for the working class, development of novel pharmacologic approaches for mental health maladies have stagnated, attributable in part to growing interest in non-pharmacological treatments (Wortzel et al. 2020), a substantial shift in funding by the National Institutes of Mental Health - the world's largest funder of mental health research-from clinical trials to basic research (Reardon 2017; Lewis-Fernandez et al. 2016) and calculated decisions by pharmaceutical companies to pursue treatment for conditions with lower risks and higher profit-margins (Miller 2010).

This confluence of historical conditions has, in part, set the stage for psychedelics to reemerge from the realm of taboo. Although these compounds remain criminalized, in recent decades one sanctioned space has continued to explore their use: clinical research. While the shaman of the Andes might look askance at the biomedical laboratories where scientists now study altered states, these clinics have become a major proving ground for the re-legitimation of psychedelics in Western culture (Garcia-Romeu et al. 2016; Reiff et al. 2020). (see Fig. 1).

In the last decade alone, governing bodies have approved clinical trials on MDMA, ketamine, LSD, psilocybin, and dimethyltryptamine-the latter two of which were also part of the Tiwanaku shaman's ancient armamentarium (Siegel 

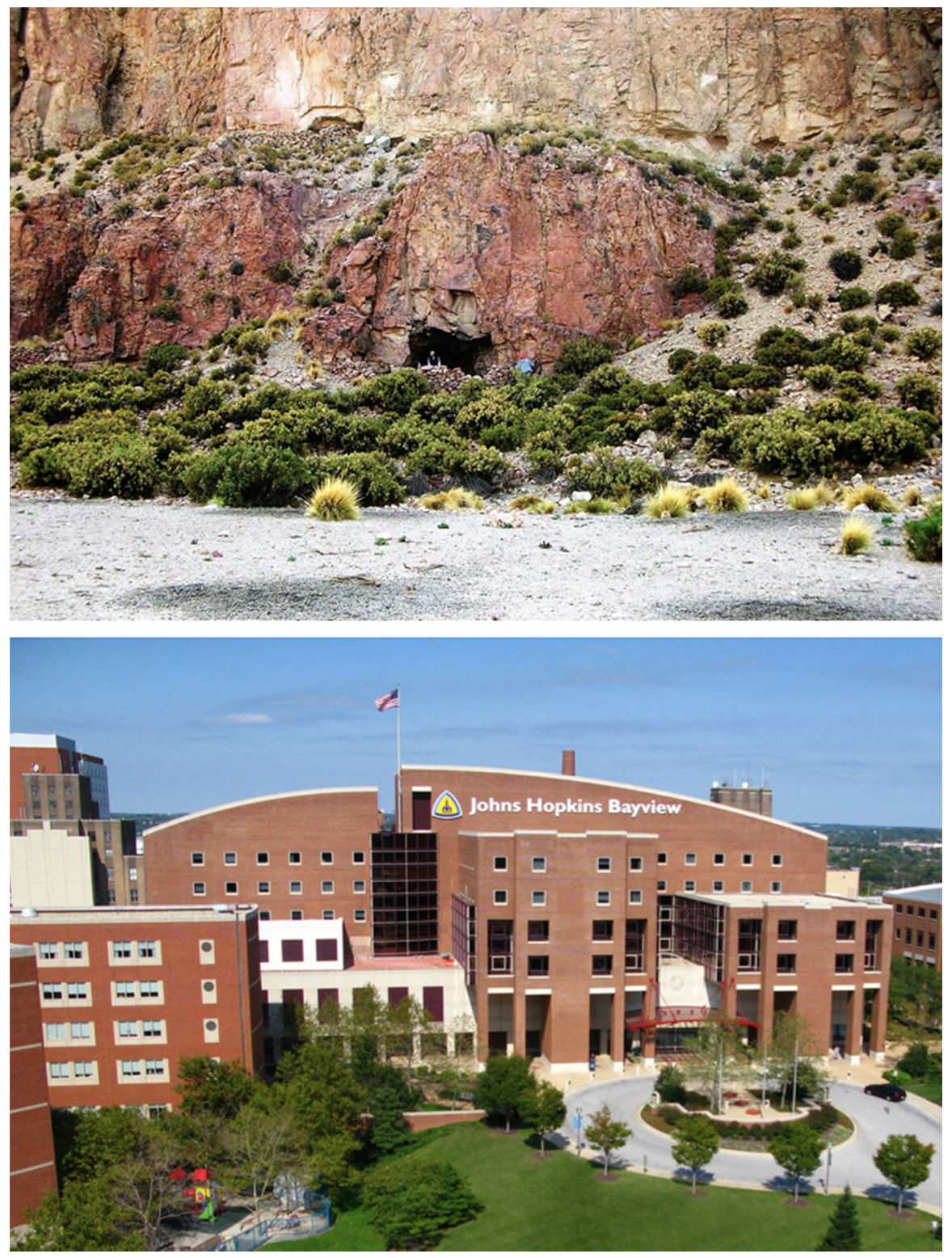

Fig. 1 Top, a cave in the Sora River valley in southwestern Bolivia where researchers discovered a 1000-year-old ritual bundle formerly belonging to a shaman of the pre-Inca Tiwanaku civilization; below, the building currently housing the Johns Hopkins Center for Psychedelic and Consciousness Research

et al. 2021). There has, in turn, been a burgeoning of peer-reviewed research establishing preliminary effectiveness of these therapies in treating depression (Carhart-Harris et al. 2021; Davis et al. 2020), substance use disorders (Bogenschutz et al. 2015; Johnson et al. 2014), PTSD (Mitchell et al. 2021), and anxiety for 
patients facing terminal cancer (Griffiths et al. 2016; Grob et al. 2011; Ross et al. 2016). New studies are beginning to examine psychedelics as potential treatments for other wide-ranging conditions such as eating disorders, migraine and cluster headaches, and early dementia (Siegel et al. 2021).

Research has concurrently sought to identify mechanisms underlying the effectiveness of such treatments. While classic psychedelics like psilocybin, LSD, and ayahuasca exert their primary effects via the serotonin $2 \mathrm{~A}\left(5-\mathrm{HT}_{2 \mathrm{~A}}\right)$ receptor that is heavily expressed throughout the cortex, other hallucinogenic compounds such as ketamine act on glutamatergic N-Methyl-D-Aspartate receptors, with both inducing neuroplastic changes that may lead to therapeutic benefits (Deyama and Duman 2020; Ly et al. 2018). Additionally, the classic psychedelics have been found to initiate a cascade of altered neural connectivity and blood-flow across brain regions including the "default-mode network" (medial prefrontal cortex, posterior cingulate cortex, inferior parietal lobule, lateral temporal cortex, hippocampus, and precuneus), amygdala, thalamus, and claustrum, disrupting established patterns of brain activity and altering a person's fundamental sense-of-self (Barrett et al. 2020; Kraehenmann et al. 2015; Preller et al. 2018; Roseman et al. 2018a,b).

Data indicate these changes correspond with the altered states that have for millennia precipitated mystical, meaningful, and transcendent experiences often characterized by profound feelings of connection, openness, and unity, and there is compelling evidence that the numinous quality of acute psychedelic experiences may itself be predictive of long-term clinical changes in mental health (Bogenschutz et al. 2015; Garcia-Romeu et al. 2014; Griffiths et al. 2016; Roseman et al. 2018a,b). Given that many mental health conditions are marked by inflexible patterns of habitual thought, feeling, and behavior (Morris and Mansell, 2018), using psychedelics to disrupt neural systems encoding such patterns provides a compelling approach for mental healthcare treatment (Carhart-Harris et al. 2014). Furthermore, the seeming ability of psychedelics to enhance cognitive, emotional, and creative flexibility, in part by evoking the mystical-type experiences long sought out by our human ancestors, can be a powerful starting point for psychological healing (Nutt and Carhart-Harris 2021; Davis et al. 2020; Mason et al. 2021; Harris, 2021). Coupled with therapeutic integration focused on insight and emotional recalibration, and in light of psychedelics' ability to promote structural and functional neural plasticity, these substances may actually help "rewire the brain" to enable long-term relief.

Indeed, beyond psychoactive compounds themselves, non-pharmacological variables have been shown to significantly impact individuals' experiences on psychedelics (Studerus et al., 2012). According to one review, "the effects of hallucinogens are heavily dependent on the expectations of the user...and the environment...in which the use takes place" (Nichols 2004, p. 137). At least with respect to psilocybin, the psychological and emotional state of the user in the 4-weeks prior to their psychedelic episode are extremely important in determining whether the subject experiences severe anxiety (i.e., a "bad trip") or has a more positive, "spiritual" encounter with what twentieth century psychologist Carl Jung famously described as the "sphere of numinous experiences" (in Hill 2013; Studerus et al. 2012). 
It is therefore notable that anthropological accounts of ayahuasca medicine in indigenous Amazonian communities often emphasize the importance of a knowledgeable and experienced curandero (healer) to act as a guide, as well as the need for days, or even weeks, of appropriate preparation in advance of actually drinking the psychedelic brew. Consider the following words of Guillermo Arrévallo, a Shipibo shaman who works in Pucallpa, Peru: "I have to organize my work around what the person accepts as their beliefs about what it is that has damaged them, the particular trauma involved. On this basis, I organize the psychological part of the person, which enables me to understand and to see the solution to their problem" (Dobkin de Rios 2005, p. 204; our emphasis). As reflected in this instance, the shaman's role has not merely been to administer a disruptive drug but to manage and reintegrate the psychological state of the patient so as to ensure the success of the healing ritual.

Similarly, the Native American Church's ritualistic use of the peyote cactus, which contains the psychoactive alkaloid mescaline, is traditionally facilitated by a shaman, or "Roadman," who guides worshippers suffering personal/familial crises through all-night prayer ceremonies in a communal tepee. This ancient practice, which is estimated to have been held for up to 40,000 years (Prue 2013), is highly ritualistic, with the initial ingestion of mescaline serving to render worshippers more open to a refiguring of maladaptive thought patterns and reinterpretation of their predicament. In the wake of inducing an altered state, the officiant leads participants in drumming, singing, praying, ceremonial tobacco use, the passing around of sacred objects, and symbolic patterns of movement around the tepee-all intended to foster a sense of transformation (Bergman 1971; Calabrese 1997). In the 1970s, American physician Robert Bergman ethnographically observed Navajo peyote use, noting the indispensable role of Roadman healers who "are trained to look after people who become excessively withdrawn...if a participant begins to stare fixedly into the fire and seems unaware of the others, the road man will speak to him and, if necessary, go to him to pray with him...fan him with an eagle feather fan, splash drops of water on him, and fan cedar incense over him" (Bergman 1971). The administration of the drug is not regarded as healing in and of itself; the therapeutic benefit requires the formation of a mentoring relationship with the healer via ritual (Prue 2013).

Contemporary clinical research has corroborated that, in contrast to using psychedelics as standalone pharmacotherapies, supportive counseling remains a crucial mediating factor (Garcia-Romeu and Richards 2018; Rucker et al. 2018). Mechanistic ingestion of a "pill" is not necessarily enough, and-in fact-may even be contraindicated. Just as general anesthesia is not prescribed for take-home use, but administered under active medical supervision and incorporated within a larger interventional context, safety and efficacy of modern psychedelic therapy seem to be optimized by similar wraparound care (Johnson et al. 2008). As we think about how to bring these treatments above-ground in an era defined by anxiety, despair, loneliness, and alienation (Case and Deaton 2020), it is constructive to imagine how mental healthcare systems might practically evolve in the future. Such an exercise seems even more crucial in the throes of a pandemic whose traumas will demand more of mental health services in years to come (Vahratian et al. 2021). 
Institutional parameters of the biomedical model will ipso facto limit how modern-day psychedelic treatment is implemented, at least initially (Johnson et al. 2008; Sessa 2005; 2016). However, one obvious question is whether Western medicine can draw upon indigenous knowledge as it integrates psychedelics into current clinical environments. It would, for instance, seem critical for health systems to preserve the ancient ritualistic elements of psychedelic-based therapies, with close attention to assessment, set and setting, time for preparation, processing and dialogue, and re-integration after profound experiences, as commonly practiced in current clinical research (Carhart-Harris et al. 2018; Garcia-Romeu and Richards 2018). Whereas studies increasingly show that a compound like ketamine may be effective when administered in standard clinical infusions (Corriger and Pickering 2019), interventions using psilocybin and MDMA have been most effective when carried out over several weeks with oversight from therapists (Carhart-Harris et al., 2021; Davis et al. 2020; Griffiths et al. 2016; Mitchell et al. 2021). Some might ask whether society is willing to invest in such resource-intensive treatments-activities for which entire days/weeks were often devoted by our ancient forebears And if so, can such interventions exist at scale within a capitalist system that often diminishes flexibility in working peoples' lives and severely strains the clinical time healers may spend with their patients? Given our growing mental health crises, it may be more apropos to ask whether we can afford to maintain an ineffectual status quo in current healthcare systems that often place profits over people.

In addition to logistical considerations, it is also worthwhile to think about clinical settings that may stand to gain most from incorporating psychedelic treatments. Research suggests a clear fit within addiction treatment and psychiatric care for people with mood or anxiety disorders, as well as facilities specializing in cancer treatment and hospice or palliative care. Could we imagine deploying psychedelics in assisted-living facilities? (George and Hanson 2019). For rehabilitation of incarcerated or formally incarcerated individuals? (Doblin 1998; Hendricks et al. 2018; Leary 1969).

Looking forward, psychedelic therapists will require systematic training and licensing consistent with our education/certification of other health professionals (Nutt and Carhart-Harris 2021; Phelps 2017). Given that an aspect of shamanic power has been the intimate "embeddedness" of the figure within the social relations of discrete human groups, it will be critical to train modern practitioners who reflect local populations and to set up equitable, culturally-sensitive treatment networks in urban and rural environments accessible to people across class, racial, gender, ethnic, and generational boundaries (i.e., not just available to a wealthy metropolitan elite) (Thrul and Garcia-Romeu 2021). How might practitioners exist as part of healthcare teams, and how accommodating will frontline professionals and funders be?

There is a long way to go in answering these and other questions, and, as recent history teaches, any paradigm-shifting will likely face major cultural and politicaleconomic resistance (Kuhn 1996). But engineering a systems that could help those who are hurting pursue the underlying causes of their pain, trauma, and distressthe mechanisms of which remain poorly understood-and enjoy lives as more open, pro-social human beings could have manifold benefits. Patients might emerge from 
therapy feeling greater sense of purpose, renewed solidarity and interdependence with others, and unity with a planet destabilized by anthropogenic climate change (Watts et al. 2017). One can imagine those successfully transformed by psychedelic therapy being less disconnected, less psychologically attached to prior internalized pain, less prone to self-harm (e.g., DoD), and less susceptible to the modern-day "altered states" offered by harmful drugs, internet, video games, social media, and mindless consumption. While admittedly speculative in the absence of researchderived findings, it is plausible that therapeutically guiding contemporary humans through psychedelic experiences could potentially serve as a means of provoking new ideas/innovations to push through areas of cultural stagnation, fragmentation, and long-standing geopolitical conflicts. For instance, the Multidisciplinary Association for Psychedelic Studies is currently pursuing psychedelic interventions for conflict resolution between Arabs/Israelis and Catholics/Protestants (Roseman et al. 2021).

It will inevitably be argued that retooling mental health systems is "too expensive". However, from a health economics perspective, potentially treating underlying causes of distress could reduce co-morbidities and ease burden on inpatient and ambulatory institutions. Moreover, as preliminary psychedelic research has established longitudinal efficacy of single-dose treatments (Griffiths et al. 2016; Ross et al. 2016), such an approach could transform current models reliant on long-term use of expensive - and often modestly effective-pharmaceuticals that commonly produce unwanted side effects. Economically, how many person-hours of work annually lost due to depression and related conditions could be recovered? Beyond economics, can we, as our ancestors before us, envision the human value of increased wellbeing, quality of life, insight, and balance and fellowfeeling that psychedelic treatments might engender?

There is much work to be done to bring such a vision to fruition. While there is emerging clinical data-for instance, a robust literature of studies establishing the safety and efficacy of ketamine-assisted treatment for depression (see Salahudeen et al. 2020) as well as increasingly high-quality studies on psilocybin-based therapies for depression (Carhart-Harris, et al. 2021; Davis et al. 2020)-larger and more robust clinical trials are needed. Emergent data will help flesh out best practices for identifying appropriate treatment candidates and proper dosages for different patient groups, optimal regimens for set/setting, and protocols for managing adverse events. Given the ancient nature of psychedelic therapies, there may be particularly important-if unconventional-roles for non-clinical experts (e.g., social scientists, archaeologists, religious studies scholars) in the clinical deployment and evaluation of modern therapies. Moreover, ethicists, philosophers, and other scholars from the social sciences and humanities may also fruitfully explore practical and ethical questions regarding the therapeutic effects of psychedelics as well as more conceptual inquiry (e.g., how the nature of the mind-brain relationship may be clarified or problematized by psychedelic therapy).

Great vigilance will be needed regarding private operators already working to commodify these complex therapies (which have been used carefully, ritualistically, with sacred purpose by humans outside of a market economy for tens of thousands of years) with quick-fix marketing hype. Importantly, increasing focus on 
psychedelics must not depoliticize larger political-economic structures that are perpetuating the material conditions actively degrading the physical and mental wellbeing of millions (Schrecker and Bambra 2015; George and Whitehouse 2021), lest psychedelics become a new "opiate of the masses" that merely distract us from rightful dissatisfaction with the systemic injustices and massive disparities in wealth, income, health and other outcomes that define our era.

Ultimately, we believe a twenty-first century "psychedelic renaissance" anchored in the lessons of antiquity can point healthcare systems-and perhaps even society itself-in a direction that is more humane, more attentive to mental health needs, and more materially (and spiritually) supportive of human flourishing and societal/planetary health in an era currently characterized by historic levels of distress and despair.

Acknowledgements None.

\section{Declarations}

Conflict of interest AGR serves as a scientific advisor to NeonMind Biosciences and ETHA Natural Botanicals. The other authors report no conflicts of interest.

Ethical approval This article does not contain any studies with human participants or animals performed by any of the authors.

\section{References}

Barrett, Frederick, Samuel Krimmel, Roland Griffiths, David Seminowicz, and Brian Mathur 2020 Psilocybin Acutely Alters the Functional Connectivity of the Claustrum with Brain Networks that Support Perception, Memory, and Attention. NeuroImage, 218:116980.

Bergman, Robert L.

1971 Navajo Peyote Use: Its Apparent Safety. American Journal of Psychiatry 128(6):695-699.

Blanchflower, David, and Andrew Oswald

2020 Trends in extreme distress in the United States, 1993-2019. American Journal of Public Health 110(10):538-1544.

Bogenschutz, Michael P., Alyssa A. Forcehimes, Jessica A. Pommy, Claire E. Wilcox, P.C.R. Barbosa, and Rick J. Strassman

2015 Psilocybin-assisted treatment for alcohol dependence: a proof-of-concept study. Journal of Psychopharmacology 29(3):289-299.

Brignone, Emily, Daniel R. George, Larry Sinoway, Curren Katz, Charity Sauder, Andrea Murray, Robert Gladden, Jennifer L. Kraschnewski. Characterizing 'Diseases of Despair' in a nationally representative sample. (2020). BMJ Open,10:e037679. doi: https://doi.org/10.1136/bmjopen-2020-037679.

Calabrese, Joseph D.

1997 Spiritual Healing and Human Development in the Native American. Psychoanlytic Review 84(2):237-255.

Carhart-Harris, Robin L., Bruna Giribaldi, Rosalind Watts, Michelle Baker-Jones, Ashleigh Murphy-

Beiner, Roberta Murphy, Jonny Martell, Allan Blemings, David Erritzoe, and David J. Nutt

2021 Trial of Psilocybin versus Escitalopram for Depression. New England Journal of Medicine 384(15):1402-1411. 
Carhart-Harris, Robin L, Leor Roseman, Eline Haijen, David Erritzoe, Rosalind Watts, Igor Branchi, and Mendel Kaelen 2018 Psychedelics and the Essential Importance of Context. Journal of Psychopharmacology, 32(7):725-731. https://doi.org/10.1177/0269881118754710.

Carhart-Harris, Robin L., Robert Leech, Peter Hellyer, Murray Shanahan, Amanda Feilding, Enzo Tagliazucci, Dante R. Chialvo, and David Nutt

2014 The entropic brain: a theory of conscious states informed by neuroimaging research with psychedelic drugs. Front. Hum. Neurosci. https://doi.org/10.3389/fnhum.2014.00020.

Carod-Artal, F.J.

2015 Hallucinogenic drugs in pre-Columbian Mesoamerican cultures. Neurología (english Edition) 30(1):42-49.

Case, Anne, and Angus Deaton 2021 Life expectancy in adulthood is falling for those without a BA degree, but as educational gaps have widened, racial gaps have narrowed. Proceedings of the National Academy of Sciences 118 (11):e2024777118. https://doi.org/10.1073/pnas.2024777118.

Case, Anne, and Angus Deaton

2020 Deaths of Despair and the Future of Capitalism. New Jersey: Princeton University Press.

Case, Anne, and Angus Deaton 2017 Mortality and Morbidity in the 21(st) Century. Brookings Pap Econ Act 397-476.

Case, Anne, and Angus Deaton

2015 Rising morbidity and mortality in midlife among white non-Hispanic Americans in the 21st century. Proceedings of the National Academy of Sciences 112:15078-15083.

Corriger, Alexandrine, and Gisele Pickering

2019 Ketamine and Depression: A Narrative Review. Drug Design, Development and Therapy $13: 3051$.

Davis., Alan K, Frederick S. Barrett, Darrick G. May, Mary P. Cosimano, Nathan D. Sepeda, Matthew W.

Johnson, Patrick H. Finan, and Roland R. Griffiths

2021 Effects of Psilocybin-Assisted Therapy on Major Depressive Disorder: A Randomized Clinical Trial. JAMA Psychiatry 78(5):481-489.

Davis, Alan K., Frederick S. Barrett, and R.R. Griffiths

2020 Psychological Flexibility Mediates the Relations Between Acute Psychedelic Effects and Subjective Decreases in Depression and Anxiety. Journal of Contextual Behavioral Science 15:39-45.

Deyama, Satoshi, and Ronald S. Duman 2020 Neurotrophic Mechanisms Underlying the Rapid and Sustained Antidepressant Actions of Ketamine. Pharmacology Biochemistry and Behavior 188:172837.

Dobkin de Rios, Marlene 1974 The Influence of Psychotropic Flora and Fauna on Maya Religion. Current Anthropology 15(2):147-164

Dobkin de Rios, Marlene

2005 Interview with Guillermo Arrévalo, a Shipibo urban shaman, by Roger Rumrrill. Journal of Psychoactive Drugs 37(2):203-207.

Doblin, Rick 1998 Dr. Leary's Concord Prison Experiment: A 34-Year Follow-up Study. Journal of Psychoactive Drugs 30(4):419-426.

Dwyer-Lindgren, Laura, Amelia Bertozzi-Villa, Rebecca Stubbs, Chloe Morozoff, Johan Mackenbach, Frank van Lenthe, Ali Mokdad, and Christopher Murray

2017 Inequalities in Life Expectancy Among US Counties, 1980 to 2014: Temporal Trends and Key Drivers. JAMA Internal Medicine 177(7):1003-1011.

Ettman, C.K., S.M. Abdalla, G.H. Cohen, L. Sampson, P.M. Vivier, and S. Galea

2020 Prevalence of Depression Symptoms in US Adults Before and During the COVID-19 Pandemic. JAMA Network Open 3(9):e2019686-e2019686.

Gaydosh, Lauren, Robert Hummer, Taylor Hargrove, Carolyn Halpern, Jon Hussey, Eric Whitsel, and Nancy Dole

2019 The Depths of Despair Among US Adults Entering Midlife. American Journal of Public Health 109:774-780.

Garcia-Romeu, Albert, and William A. Richards

2018 Current Perspectives on Psychedelic Therapy: Use of Serotonergic Hallucinogens in Clinical Interventions. International Review of Psychiatry 30(4):291-316.

Garcia-Romeu, Albert, Brennan Kersgaard, and Peter H. Addy

2016 Clinical Applications of Hallucinogens: A Review. Experimental and Clinical Psychopharmacology 24(4):229. 
Garcia-Romeu, Albert, Roland Griffiths, and Matthew W. Johnson

2014 Psilocybin-Occasioned Mystical Experiences in the Treatment of Tobacco Addiction. Current Drug Abuse Reviews 7(3):157-164.

George, Daniel R., and Peter J. Whitehouse

2021 American Dementia: Brain Health in an Unhealthy Society. Baltimore: Johns Hopkins Press; In Press.

George, Daniel R., Snyder, Bethany, Lauren Van Scoy, Emily Brignone, Lawrence Sinoway, Charity Sauder, Andrea Murray, Robert Gladden, Shayann Ramedani, Alana Ernharth, Neha Gupta, Savreen Saran, and Jennifer Kraschnewski 2021 Perceptions on Diseases of Despair by Members of Rural and Urban High-Prevalence Communities: A Qualitative Study. JAMA Network Open 4(7):e2118134.

George, Daniel R., and Ryan Hanson

2019 Imagining a Role for Psychedelics in Dementia Care. American Journal of Geriatric Psychiatry 27(9):1028-1030.

Griffiths, Roland R., Matthew W. Johnson, Michael A. Carducci, Annie Umbricht, William A. Richards,

Brian D. Richards, Mary P. Cosimano, and Margaret A. Klinedinst

2016 Psilocybin Produces Substantial and Sustained Decreases in Depression and Anxiety in Patients with Life-Threatening Cancer: A Randomized Double-Blind Trial. Journal of Psychopharmacology 30(12):1181-1197.

Grob, Charles A., Alicia L. Danforth, Gurpreet S. Chopra, Marycie Hagerty, Charles R. McKay, Adam L.

Halberstadt, and George R. Greer

2011 Pilot Study of Psilocybin Treatment for Anxiety in Patients with Advanced-Stage Cancer. Archives of General Psychiatry 68(1):71-78.

Harris, James C. (2021). Psychedelic-Assisted Therapy and Carl Jung's Red Book. JAMA Psychiatry, published online, June 16: doi:https://doi.org/10.1001/jamapsychiatry.2021.1207

Hendricks, Peter S., Michael Scott Crawford, Karen L. Cropsey, N. Heith Copes, Wiles Sweat, Zach Walsh, and Gregory Pavela

2018 The Relationships of Classic Psychedelic Use with Criminal Behavior in the United States Adult Population. Journal of Psychopharmacology 32(1):37-48.

Hill S.J. 2013 Confrontation with the Unconscious: Jungian Depth Psychology and Psychedelic. Muswell Hill Press

Johnson, Matthew W., Albert Garcia-Romeu, M.P. Cosimano, and Roland R. Griffiths 2014 Pilot Study of the 5-HT2AR Agonist Psilocybin in the Treatment of Tobacco Addiction. Journal of Psychopharmacology 28(11):983-992

Johnson, Matthew W., W.A. Richards, and Roland R. Griffiths

2008 Human Hallucinogen Research: Guidelines for Safety. Journal of Psychopharmacology 22(6):603-620.

Kraehenmann, Rainer, Katrin Preller, Milan Scheidegger, Thomas Pokorny, Oliver Bosch, Erich Seifritz, and Franz Vollenweider

2015 Psilocybin-Induced Decrease in Amygdala Reactivity Correlates with Enhanced Positive Mood in Healthy Volunteers. Biological Psychiatry 78(8):572-581.

Kuhn, Thomas S.

1996 The Structure of Scientific Revolutions. 3rd Edition. Chicago, IL: University of Chicago Press.

Leary, $\mathrm{T}$.

1969 The Effects of Consciousness-Expanding Drugs on Prisoner Rehabilitation. Psychedelic Review 10:29-45.

Lewis-Fernández, Roberto, Mary Jane Rotheram-Borus, Virginia T. Betts, Lisa Greenman, Susan M. Essock, Javier I. Escobar, Deanna Barch, Michael F. Hogan, Patricia A. Arean, Benjamin Druss, Ralph DiClemente, Thomas H. McGlashan, Dilip V. Jeste, Enola K. Proctor, Pedro Ruiz, John Rush, Glorisa J.

Canino, Carl C. Bell, Renata Henry, and P. Portia Iversen

2016 Rethinking Funding Priorities in Mental Health Research. British Journal of Psychiatry 208(6):507-509. https://doi.org/10.1192/bjp.bp.115.179895.

Lim G.Y., W.W. Tam, Y. Lu, C.S. Ho, M.W. Zhang, R.C. Ho 2018 Prevalence of Depression in the Community from 30 Countries between 1994 and 2014. Scientific Reports 8(1):2861.

Ly, Calvin, Alexandra Greb, Lindsay Cameron, Jonathan Wong, Eden Barragan, Paige Wilson, Kyle Burbach, Sina Zarandi, Alexander Sood, Michael Paddy, Whitney Duim, A Megan Dennis, Kimberly McAllister, Kassandra Ori-McKenney, John Gray, and David Olson

2018 Psychedelics Promote Structural and Functional Neural Plasticity. Cell Reports 23(11):31703182 . 
Marmot, Michael

2018 Social Causes of the Slowdown in Health Improvement. Journal of Epidemiology and Community Health 72:359-360.

DaRios, B., S.W. Toennes, P. Stiers, A. Fielding, and J.G. Raemaekers

2021 Spontaneous and Deliberate Creative Cognition During and After Psilocybin Exposure. Transl Psychiatry 11:209. https://doi.org/10.1038/s41398-021-01335-5.

Miller, Melanie, Juan Albarracin-Jordan, Christine Moore, and Jose Capriles

2019 Chemical Evidence for the Use of Multiple Psychotropic Plants in a 1,000-Year-Old Ritual Bundle from South America. Proceedings of the National Academy of Sciences 116(23):1120711212.

Miller, Greg

2010 Is Pharma Running Out of Brainy Ideas?. Science 329(5991):502-504. https://doi.org/ 10.1126/science.329.5991.502; PMID: 20671165.

Mitchell, Jennifer M., Michael Bogenschutz, Alia Lilienstein, Charlotte Harrison, Sarah Kleiman, Kelly Parker-Guilbert, Marcela Ot'alora G., Wael Garas, Casey Paleos, Ingmar Gorman, Christopher Nicholas, Michael Mithoefer, Shannon Carlin, Bruce Poulter, Ann Mithoefer, Sylvestre Quevedo, Gregory Wells, Sukhpreet S. Klaire, Bessel van der Kolk, Keren Tzarfaty, Revital Amiaz, Ray Worthy, Scott Shannon, Joshua D. Woolley, Cole Marta, Yevgeniy Gelfand, Emma Hapke, Simon Amar, Yair Wallach, Randall Brown, Scott Hamilton, Julie B. Wang, Allison Coker, Rebecca Matthews, Alberdina de Boer, Berra Yazar-Klosinski, Amy Emerson, and Rick Doblin. 2021 MDMA-Assisted Therapy for Severe PTSD: A Randomized, Double-Blind, Placebo-Controlled Phase 3 Study. Nature Medicine 1-9. Morris, L., and W. Mansell

2018 A Systematic Review of the Relationship Between Rigidity/Flexibility and Transdiagnostic Cognitive and Behavioral Processes that Maintain Psychopathology. Journal of Experimental Psychopathology 9(3):2043808718779431.

Murphy, Sherry, Jiaquan Xu, Kenneth Kochanek, and Elizabeth Arias. (2018). Mortality in the United States, 2017. NCHS Data Brief, No 328. Hyattsville, MD: National Center for Health Statistics.

Nichols, David E.

2004 Hallucinogens. Pharmacology \& Therapeutics 101(2):131-181.

Nutt, David J., and Robin Carhart-Harris

2021 The Current Status of Psychedelics in Psychiatry. JAMA Psychiatry. 78(2):121-122.

Nutt, David J., Leslie A. King, and David E. Nichols

2013 Effects of Schedule I Drug Laws on Neuroscience Research and Treatment Innovation. Nature Reviews Neuroscience 14(8):577-585.

Ouvaroff, Sergay S. 1817 Essay on the Mysteries of Eleusis. London: Rodwell and Martin (Reprint: United States: Kessinger Publishing, 2004).

Phelps, Janis

2017 Developing Guidelines and Competencies for the Training of Psychedelic Therapists. Journal of Humanistic Psychology 57(5):450-487.

Preller, Katrin, Joshua Burt, Lisa Jie, Charles Schliefer, Brendan Adkinson, Philipp Stampfli, Erich Seifritz, Grega Repovs, John Krystal, John Murray, Franz Vollenweider, and Alan Anticevic 2018 Changes in Global and Thalamic Brain Connectivity in LSD-Induced Altered States of Consciousness are Attributable to the 5-HT2A Receptor. Elife 7:e35082.

Prue, Bob

2013 Indigenous Supports for Recovery from Alcoholism and Drug Abuse: The Native American Church. Journal of Ethnic \& Cultural Diversity in Social Work 22:271-287.

Reardon, Sara

2017 US Mental-Health Agency's Push for Basic Research Has Slashed Support for Clinical Trials. Nature 546:339. https://doi.org/10.1038/546338a.

Reiff, Collin M., Elon E. Richman, Charles B. Nemeroff, Lina L. Carpenter, Alik S. Widge, Carolyn I. Rodriguez, Ned H. Kalin, and William M. McDonald 2020 Work Group on Biomarkers and Novel Treatments, a Division of the American Psychiatric Association Council of Research. Psychedelics and psychedelic-assisted psychotherapy. American Journal of Psychiatry 177(5):391-410.

Ren, Meng, Zihua Tang, Xinhua Wu, Robert Spengler, Hongen Jiang, Yimin Yang, and Nicole Boivin 2019 The Origins of Cannabis Smoking: Chemical Residue Evidence from the First Millennium BCE in the Pamirs. Science Advances 5(6):eaaw1391.

Roseman, Leor, Yiftach Ron, Antwan Saca, Natalie Ginsberg, Lisa Luan, Nadeem Karkabi, Rick Doblin, and Robin Carhart-Harris 
2021 Relational Processes in Ayahuasca Groups of Palestinians and Israelis. Frontiers in Pharmacology $12: 300$.

Roseman, Leor, Lysia Demetriou, Matthew Wall, David J. Nutt, and Robin Carhart-Harris

2018 Increased Amygdala Responses to Emotional Faces After Psilocybin for Treatment-Resistant Depression. Neuropharmacology 142:263-269.

Roseman, Leor, David J. Nutt, and Robin Carhart-Harris

2018 Quality of Acute Psychedelic Experience Predicts Therapeutic Efficacy of Psilocybin for Treatment-Resistant Depression. Frontiers of Pharmacology. https://doi.org/10.3389/ fphar.2017.00974.

Ross, Stephen, Anthony Bossis, Jeffret Guss, Gabrielle Agin-Liebes, Tara Malone, Barry Cohen, Sarah E. Mennenga, Alexander Belser, Krystallia Kalliontzi, James Babb, Su Zhe, Patricia Corby, and Brian L. Schmidt

2016 Rapid and Sustained Symptom Reduction Following Psilocybin Treatment for Anxiety and Depression in Patients with Life-Threatening Cancer: A Randomized Controlled Trial. Journal of Psychopharmacology 30(12):1165-1180.

Rucker, James J.H., Jonathan Iliff, and David J. Nutt

2018 Psychiatry \& the Psychedelic Drugs. Past, Present \& Future. Neuropharmacology 142:200-218.

Salahudeen, Mohammed S., Cameron M. Wright, and Gregory M. Peterson

2020 Esketamine: New Hope for the Treatment of Treatment-Resistant Depression? A Narrative Review. Therapeutic Advances in Drug Safety 11:2042098620937899. https://doi.org/10.1177/ 2042098620937899.

Samorini, Giorgio

2019 The Oldest Archeological Data Evidencing the Relationship of Homo Sapiens with Psychoactive Plants: A Worldwide Overview. Journal of Psychedelic Studies 3(2):63-80.

Ted, Schrecker, and Clare Bambra

2015 How Politics Makes us Sick: Neoliberal Epidemics. New York: Palgrave Macmillan.

Scutchfield, Douglas F., and William Keck

2017 Deaths of Despair: Why? What to Do?. American Journal of Public Health 107:1564-1565.

Sessa, Ben

2005 Can Psychedelics have a Role in Psychiatry Once Again?. The British Journal of Psychiatry 186(6):457-458.

Sessa, Ben 2016 The History of Psychedelics in Medicine. Handbuch Psychoaktive Substanzen. Springer

Reference Psychologie. Berlin: Springer.

Shiels, Meredith, Zaria Tatalovich, Yingxi Chen, Emily Haozous, Patricia Hartge, Anna Napoles, Eliseo

Perez-Stable, Erik Rodriquez, Susan Spillane, David Thomas, Diana Withrow, Amy Berrington, and AB de Gonzalez

2020 Trends in Mortality From Drug Poisonings, Suicide, and Alcohol-Induced Deaths in the United States From 2000 to 2017. JAMA Network Open 3(9):e2016217. https://doi.org/10.1001/ jamanetworkopen.2020.16217.

Siegel, Ashley N., Shakila Meshkat, Katie Benitah, Orly Lipsitz, Hartej Gill, Leanna M.W. Lui, Kayla M.

Teopiz, Roger S. McIntyre, and Joshua D. Rosenblat

2021 Registered Clinical Studies Investigating Psychedelic Drugs for Psychiatric Disorders. Journal of Psychiatric Research 139:71-81.

Smeraldo Schell, Kait, and Jennifer Silva 2020 Resisting Despair: Narratives of Disruption and Transformation among White Working-Class Women in a Declining Coal-Mining Community. Gender \& Society 10:0891243220948218

Staal, Frits

2001 How a Psychoactive Substance Becomes a Ritual: The Case of Soma. Social Research 68:745778.

Stein, Elizabeth, Keith Gennuso, Donna Ugboaja, and Patrick Remington

2017 The Epidemic of Despair Among White Americans: Trends in the Leading Causes of Premature Death, 1999-2015. American Journal of Public Health 107:1541-1547.

Studerus, Erich, Alex Gamma, Michael Kometer, and Franz X. Vollenweider 2012 Prediction of Psilocybin Response in Healthy Volunteers. PLoS ONE 7(2):e30800.

Thrul, Johannes, and Albert Garcia-Romeu 2021 Whitewashing Psychedelics: Racial Equity in the Emerging Field of Psychedelic-Assisted Mental Health Research and Treatment. Drugs: Education, Prevention and Policy 28(3):211-214. https://doi.org/10.1080/09687637.2021.1897331. 
Vahratian, Anjel, Stephen J. Blumberg, Emily P. Terlizzi, Jeanine S. Schiller 2021 Symptoms of Anxiety or Depressive Disorder and Use of Mental Health Care Among Adults During the COVID-19 Pandemic - United States, August 2020-February 2021. MMWR Morb Mortal Wkly Rep 70:490-494. https://doi.org/10.15585/mmwr.mm7013e2external icon

Walter, Mariko N., and Eva Jane Neumann Fridman. (Eds.). 2004 Shamanism: An encyclopedia of world beliefs, practices, and culture (Vol. 1). Abc-clio.

Wasson, Gordan R., Carl A.P. Ruck, and Albert Hofmann

1978 The Road to Eleusis: Unveiling the Secret of the Mysteries. Brace, Jovanovich: Harcourt.

Watts, Rosalind, Camilla Day, Jacob Krzanowski, David J. Nutt, and Robin Carhart-Harris

2017 Patients' Accounts of Increased "Connectedness" and "Acceptance" After Psilocybin for Treatment-Resistant Depression. Journal of Humanistic Psychology 57(5):520-564.

Well Being Trust 2020 Pain in the Nation: Building a National Resilience Strategy. Alcohol and Drug Misuse and Suicide and the Millennial Generation—a Devastating Impact. https://wellbeingtrust.org/ wp-content/uploads/2019/06/TFAH-2019-YoundAdult-Pain-Brief-FnlRv.pdf

Well Being Trust 2021 Pain in the Nation: Alcohol, Drug, and Suicide Epidemics: Special Feature: COVID-19 and Trauma. https://wellbeingtrust.org/wp-content/uploads/2021/05/2021PainInTheNation-FINAL-May-12.pdf

Woolf, Steven, and Heidi Schoomaker

2019 Life Expectancy and Mortality Rates in the United States, 1959-2017. Journal of the American Medical Association 322(20):1996-2016.

Wortzel, Joshua R., Brandon E. Turner, Brannon T. Weeks, Christopher Fragassi, Virginia Ramos, Thanh Truong, Desiree Li, Omar Sahak, and Hochang Benjamin Lee. 2020 Trends in Mental Health Clinical Research: Characterizing the ClinicalTrials.gov registry from 2007-2018. PLoS ONE 15(6):e0233996. https://doi.org/10.1371/journal.pone.0233996

Publisher's Note Springer Nature remains neutral with regard to jurisdictional claims in published maps and institutional affiliations. 